Saudi Journal of Biomedical Research

Abbreviated Key Title: Saudi J Biomed Res ISSN 2518-3214 (Print) |ISSN 2518-3222 (Online)

\title{
Cerebrospinal Fluid Glutamic Oxaloacetic Transaminase and Lactate Dehydrogenase Levels in Alzheimer's disease
}

\author{
Subhramay Chatterjee ${ }^{1}$, Sandip Chakraborti ${ }^{2 *}$ \\ ${ }^{1}$ Associate Professor, Biochemistry Department, Murshidabad Medical College and Hospital Berhampore, West Bengal, India \\ ${ }^{2}$ Associate Professor, Biochemistry Department, NRS Medical College and Hospital Kolkata, West Bengal, India
}

DOI: $10.36348 /$ sjbr.2020.v05i01.002

| Received: 16.01 .2020 | Accepted: 23.01.2020 | Published: 25.01 .2020

*Corresponding author: Sandip Chakraborti

\section{Abstract}

Glutamic oxaloacetic transaminase and lactate dehydrogenase are present in brain. The present study was undertaken to investigate whether there was any change in cerebrospinal fluid glutamic oxaloacetic transaminase and/or lactate dehydrogenase levels in Alzheimer's disease. In the present case control study cerebrospinal fluid glutamic oxaloacetic transaminase and lactate dehydrogenase levels were assayed in 162 Alzheimer's disease cases and 149 healthy controls. Glutamic oxaloacetic transaminase levels of cases were highly significantly increased with respect to the levels of controls. Lactate dehydrogenase levels of cases were not significantly increased with respect to the levels of controls. Data from the present study might help in understanding the biochemical basis of Alzheimer's disease. It is stressed that these findings should be supplemented by further prospective research.

Keywords: Alzheimer's disease, cerebrospinal fluid, glutamic oxaloacetic transaminase, lactate dehydrogenase.

Copyright @ 2020: This is an open-access article distributed under the terms of the Creative Commons Attribution license which permits unrestricted use, distribution, and reproduction in any medium for non-commercial use (NonCommercial, or CC-BY-NC) provided the original author and source are credited.

\section{INTRODUCTION}

Alzheimer's disease (AD) is the most common cause of dementia, and may be involved in as many as $80 \%$ of cases [1]. It typically presents with a progressive loss of cognitive function and memory [2]. This symptom occurs because the first neurons to die and malfunction are usually neurons in brain regions involved in forming new memories. There are also characteristic multiple depositions of $\beta$ A4-amyloid in brains of Alzheimer patients. Although research has revealed a great deal about $\mathrm{AD}$, the precise changes in the brain that trigger the development of $\mathrm{AD}$, and the order in which they occur, largely remain unknown [3]. Currently, there are no treatments that prevent or slow the disease. Biomarkers that can detect AD pathology in its early stages and predict dementia progress may have significant utility in clinical trial design, providing greater diagnostic certainty for enrollment than is possible by clinical diagnosis alone, and allowing for the selection of individuals at greater risk of developing more cognitive impairment in the future, ultimately resulting in trials of shorter duration, smaller size, and reduced cost. Novel biomarkers should also allow the design of early mechanism-based therapeutic intervention.
Glutamic oxaloacetic transaminase (GOT), an enzyme, is present in high concentration in a number of animal tissues including brain [4]. Lactate dehydrogenase (LDH), another enzyme which reversibly converts lactate to pyruvate has five isoenzymes, which are tetramers formed from various combinations of two types of subunits $[5,6]$. One of the isoenzymes of LDH also is expressed in brain.

The present study was undertaken to investigate whether there was any change in CSF (Cerebrospinal fluid) GOT and/or LDH levels in AD, to facilitate the search for a parameter that may help in understanding biochemical and pathophysiologic changes in AD brain.

\section{MATERIALS AND METHODS}

This study was a hospital-based, case-control study conducted in the Department of Biochemistry of a medical college and hospital in West Bengal, India. The study was approved by the local ethical committee and all cases and controls gave their informed consent to take part in this investigation. 
The duration of the present study was 11 months and included $162 \mathrm{AD}$ patients (group 1) attending the outpatient department, duration ranging from 2 months to 9 months? In addition, 149 patients (group 2), who were age- and sex-matched with the AD subjects, served as controls. The controls had attended the OPD with minor ailments unrelated to $\mathrm{AD}$. Complete history and physical examination of all cases and controls were undertaken. Exclusion criteria included smokers and alcoholics, patients taking certain drugs or with history of trauma or any surgery within 1 month prior to sampling, and those suffering from systemic diseases or any malignancy. Lastly, subjects having kidney, lungs, pancreas, muscle, liver dysfunction, brain diseases other than $\mathrm{AD}$ and blood disorders like hemolytic anemia, low platelet count were also excluded.

CSF was collected from each case and control. All samples were coded and assayed in a blind fashion by an investigator who was unaware of the subjects' clinical status. GOT and LDH were assayed using commercial kit (Siemens, Mumbai, India) and Coralab 3000 semiautoanalyser.

Statistical analysis of the data was performed by using Statistical Package for Social Sciences and inferences were drawn. $\mathrm{p}<0.05$ was considered to be significant and $\mathrm{p}<0.001$ highly significant.

\section{RESULTS}

170 subjects were selected for both groups (1 and 2), but 8 and 21 subjects dropped out, so that finally the number of subjects in groups 1 and 2 stabilized at 162 and 149 respectively.

Table-1: CSF GOT and LDH levels, as Mean \pm SD,

\begin{tabular}{|l|l|l|}
\multicolumn{1}{|c|}{ (in IU/L) in cases and controls } \\
\hline & GOT & LDH \\
\hline Group 1 & $47.2 \pm 3.1$ & $38.3+4.8$ \\
\hline Group 2 & $34.9 \pm 1.8$ & $37.1+6.2$ \\
\hline
\end{tabular}

\section{For SGOT}

GOT levels of group 1 were highly significantly increased with respect to GOT levels of group $2(\mathrm{p}<0.0001)$.

Number of subjects in groups 1 and 2 were 162 and 149 respectively. 95\% confidence interval of the difference of the means of groups 1 and 2: from 11.728 to 12.872

Test Statistic $t=42.3113$, degree of freedom is 309; standard error of difference is 0.291. SEM of groups 1 and 2 are 0.244 and 0.147 respectively.

\section{For LDH}

LDH levels of group 1 were not significantly increased with respect to LDH levels of group 2 $(\mathrm{p}=0.0562)$.
Number of subjects in groups 1 and 2 were 162 and 149 respectively.

$95 \%$ confidence interval of the difference of the means of groups 1 and 2: from -0.032 to 2.432

Test Statistic $\mathrm{t}=1.9169$, degree of freedom is 309 , standard error of difference is 0.626 .

SEM of groups 1 and 2 are 0.377 and 0.508 respectively.

\section{DISCUSSION}

GOT levels in CSF might improve the diagnosis of $\mathrm{AD}$ dementia in combination with tau [7]. In a sample of 142 subjects with mild cognitive impairment (early stage of AD) it was found that three proteins including GOT were able to significantly predict cognitive decline .But these associations were mild and no protein remained significant after multiple comparison correction. Some proteins including GOT were found to be related to a longitudinal change in Mini-mental state examination score over a 4 year period. Again, the statistical effects associated with this finding were mild and no result passed multiple comparison correction [8].

Kaiser et al. found that older subjects had lower glutamate concentration in the motor cortex and higher glutamine concentration in corona radiata, compared to younger subject [9]. Several studies have found reduced expression of Glutamine synthetase (GS) in $\mathrm{AD}[10,11]$ Also, in the present study, GOT levels of AD patients were highly significantly increased with respect to GOT levels of controls (table 1). GOT catalyzes the reversible transamination reaction by which glutamate and oxaloacetate interact to form aspartate and $\alpha$-ketoglutarate. Glutamine synthetase catalyzes the interconversion of glutamate and glutamine. Combining evidence from the above mentioned data, in our opinion, increased GOT levels converts more glutamate to $\alpha$-ketoglutarate, and reduced levels of GS does not convert glutamine to glutamate. As a result, glutamate levels are decreased in motor cortex and glutamine levels are higher in corona radiata.

Neuropathology and structural MRI studies have pointed to the medial temporal lobe (MTL) as the brain region earliest affected in AD. Fluoro-2-deoxy-Dglucose positron emission tomography (FDG-PET) imaging has revealed glucose metabolic reductions in the parieto-temporal, frontal and posterior cingulate cortices to be the hallmark of $\mathrm{AD}$. Moreover, recent MRI-guided FDG-PET studies have shown that MTL hypometabolism is the most specific and sensitive measure for the identification of mild cognitive impairment of AD [12]. So, in our opinion, to compensate for reduced metabolism of glucose, glucogenic aminoacids may be used as alternative sources of energy. This, in turn can be another reason 
for increased activity of aminotransferases including GOT.

In the present study, LDH levels of cases were not significantly increased with respect to LDH levels of controls (table 1). This is in accordance with the study by Chandrashekharan et al. who found that the amount of nuclear-encoded lactate dehydrogenase B mRNA in brain was not altered with respect to normal control brains [13]. This finding also supports the works of Bigl et al. where no interaction was detectable between lactate dehydrogenase and $\beta$ A4-amyloid depositions in brain [14].

Limitations of this study are that the number of patients included in the study was relatively small. Further, the study population underwent only one CSF sample measurement, which may raise discussion about the reproducibility of the results. But repeated sampling entails more patient discomfort, risk, ethical considerations and complications, and patient acceptance would have been lower, resulting in patient fallout. At this point it should be noted that the patient selection did not reflect the whole actual population. Obviously, extrapolation of our findings into other populations with $\mathrm{AD}$ might be under scrutiny, since $\mathrm{AD}$ may be genetically oriented and various expressions of the related polymorphisms may be involved. So, work with more number of subjects can help in understanding the insights of this disease. Nonetheless, this study presents some important findings and the authors believe that these findings taken together might help in unravelling some data unexplained so far in AD.

\section{CONCLUSION}

Findings from the present study would be beneficial to the scientific community, but it is stressed that these should be supplemented by further prospective research with larger numbers of the patients. If the results can be confirmed a promising diagnostic or prognostic biomarker can be evolved to facilitate clinical trial designs.

\section{REFERENCES}

1. Birks, J. S., \& Harvey, R. J. (2018). Donepezil for dementia due to Alzheimer's disease. Cochrane Database of systematic reviews, (6).

2. Guerreiro, R., Wojtas, A., Bras, J., Carrasquillo, M., Rogaeva, E., Majounie, E., \& Hazrati, L. (2013). TREM2 variants in Alzheimer's disease. New England Journal of Medicine, 368(2), 117-127.

3. Alzheimer's Association, Thies, W., \& Bleiler, L. (2013). 2013 Alzheimer's disease facts and figures. Alzheimer's \& dementia, 9(2), 208-245.
4. CHINSKY, M., \& SHERRY, S. (1957). Serum transaminase as a diagnostic aid. AMA archives of internal medicine, 99(4), 556-568.

5. Gay, R. J., McComb, R. B., \& Bowers, G. N. (1968). Optimum reaction conditions for human lactate dehydrogenase isoenzymes as they affect total lactate dehydrogenase activity. Clinical chemistry, 14(8), 740-753.

6. Boyer, S. H., Fainer, D. C., \& Watson-Williams, E. J. (1963). Lactate dehydrogenase variant from human blood: evidence for molecular subunits. Science, 141(3581), 642-643.

7. Guo, L. H., Alexopoulos, P., Wagenpfeil, S., Kurz, A., Perneczky, R., \& Alzheimer's Disease Neuroimaging Initiative. (2013). Plasma proteomics for the identification of Alzheimer's disease. Alzheimer disease and associated disorders, 27(4).

8. Khan, W., Aguilar, C., Kiddle, S. J., Doyle, O., Thambisetty, M., Muehlboeck, S., \& Giampietro, V. (2015). A subset of cerebrospinal fluid proteins from a multi-analyte panel associated with brain atrophy, disease classification and prediction in Alzheimer's disease. PloS one, 10(8), e0134368.

9. Kaiser, L. G., Schuff, N., Cashdollar, N., \& Weiner, M. W. (2005). Age-related glutamate and glutamine concentration changes in normal human brain: $1 \mathrm{H}$ MR spectroscopy study at 4 T. Neurobiology of aging, 26(5), 665-672.

10. Le Prince, G., Delaere, P., Fages, C., Lefrançois, T., Touret, M., Salanon, M., \& Tardy, M. (1995). Glutamine synthetase (GS) expression is reduced in senile dementia of the Alzheimer type. Neurochemical research, 20(7), 859-862.

11. Butterfield, D. A., Hensley, K., Cole, P., Subramaniam, R., Aksenov, M., Aksenova, M., ... \& Carney, J. M. (1997). Oxidatively induced structural alteration of glutamine synthetase assessed by analysis of spin label incorporation kinetics: relevance to Alzheimer's disease. Journal of neurochemistry, 68(6), 2451-2457.

12. Mosconi, L. (2005). Brain glucose metabolism in the early and specific diagnosis of Alzheimer's disease. European journal of nuclear medicine and molecular imaging, 32(4), 486-510.

13. Chandrasekaran, K., Giordano, T., Brady, D. R., Stoll, J., Martin, L. J., \& Rapoport, S. I. (1994). Impairment in mitochondrial cytochrome oxidase gene expression in Alzheimer disease. Molecular brain research, 24(1-4), 336-340.

14. Bigl, M., \& Eschrich, K. (1995). Interaction of rat brain phosphofructokinase with Alzheimer's $\beta$ A4amyloid. Neurochemistry international, 26(1), 6975. 